\title{
Do diabetes, metabolic syndrome or their association equally affect biventricular function? A tissue Doppler study
}

\author{
Francesco Paneni ${ }^{1}$, Mario Gregori ${ }^{2}$, Giuliano Tocci $^{1}$, Francesca Palano ${ }^{2}$, Giuseppino Massimo Ciavarella ${ }^{2}$, \\ Giulia Pignatelli ${ }^{2}$, Andrea Marra ${ }^{2}$, Sebastiano Sciarretta ${ }^{1}$, Andrea Ferrucci ${ }^{2}$ and Massimo Volpe ${ }^{1,2}$
}

Metabolic syndrome (MetS) and type 2 diabetes (T2DM) have been associated with an impairment of left (LV) and right ventricular (RV) function as well as an increased risk of heart failure (HF). However, it remains unclear whether these clinical entities or their associations promote a similar derangement of biventricular function. Overall, $\mathbf{3 4 5}$ patients without overt cardiovascular disease consecutively underwent routine blood chemistry including high-sensitivity C reactive protein (hs-CRP) and echocardiographical examination with conventional and tissue Doppler imaging (TDI) of both ventricles. According to the ATP III criteria and fasting glucose levels, the study population was stratified into four groups: (1) healthy controls $(n=120)$; (2) MetS without T2DM ( $n=84)$; (3) T2DM without MetS $(n=49)$; and (4) MetS + T2DM $(n=92)$. The Myocardial performance index (MPI) of the RV and LV was obtained with a multi-segmental approach using TDI. Patients with MetS and T2DM exhibited a similar impairment of biventricular function compared with healthy controls, whereas a further decline was observed in patients having both MetS and T2DM. In addition to MetS markers, hs-CRP exhibited the strongest association with the MPI of both ventricles. Regression analyses indicated that individual MetS markers were inferior to MetS in identifying subtle cardiac dysfunction. Independent associations of MetS and T2DM with biventricular dysfunction were comparable, and the coexistence of MetS and T2DM exhibited the highest risk for biventricular dysfunction. Our findings emphasize the importance of MetS as an equivalent of T2DM and support a synergic effect of these clinical conditions on cardiac organ damage requiring more aggressive therapeutic strategies to prevent HF. Hypertension Research (2013) 36, 36-42; doi:10.1038/hr.2012.137; published online 6 September 2012

Keywords: biventricular dysfunction; diabetes; heart failure; metabolic syndrome; tissue Doppler imaging

\section{INTRODUCTION}

Type 2 diabetes (T2DM) is recognized as a strong predictor of heart failure (HF), regardless of the presence of other cardiovascular risk factors. The annual incidence of HF in the diabetic population ranges from 14 to 31 out of every 1000 individuals per year, and the reported relative risk varies from 1.8 to $3.1 .^{1-3}$ Fasting blood glucose and glycosylated hemoglobin have been strongly associated with the magnitude of HF, with a reported $8 \%$ increase in the risk of $\mathrm{HF}$ for every $1 \%$ increase in HbAlc. ${ }^{4}$ More recently, the metabolic syndrome (MetS), an insulin-resistant state defined by a cluster of cardiovascular risk factors, has been associated with myocardial fibrosis and stiffness leading to left ventricular (LV) dysfunction and an increased risk of HF over time. ${ }^{5-7}$ However, other evidence indicates that the risk of HF in patients with MetS is largely inferior compared with the risk for patients with T2DM, and recent studies do not confirm an independent association between MetS and HF occurrence. ${ }^{8}$
Different factors may precipitate chamber dysfunction in T2DM and MetS. In diabetic patients, hyperglycemia activates critical pathways leading to myocardial inflammation and fibrosis. By contrast, insulin resistance represents the main pathological substrate sustaining myocardial dysfunction in patients with MetS. Despite conflicting evidence concerning the association of MetS and T2DM with cardiac dysfunction, available recommendations consider these two clinical entities in the same risk category. ${ }^{9}$ Indeed, both T2DM and MetS are labeled as 'high risk' conditions even in the absence of studies that clearly support a comparable preclinical effect on cardiac structure and function. In this regard, studies designed to appraise the independent contribution of MetS, T2DM or their combinatorial effect on biventricular function are lacking.

In this study, we investigate the impact of T2DM, MetS and their association on indices of left and right ventricular (RV) function in a cohort of consecutive patients without overt cardiovascular disease.

IIRCCS Neuromed, Pozzilli, Italy and 2Department of Clinical and Molecular Medicine, Division of Cardiology, University of Rome 'Sapienza,' Sant'Andrea Hospital, Rome, Italy Correspondence: Professor M Volpe, Department of Clinical and Molecular Medicine, Division of Cardiology, University of Rome 'Sapienza,' Sant'Andrea Hospital, Via di Grottarossa, Rome 1035-1039, Italy.

E-mail: massimo.volpe@uniroma1.it

Received 15 February 2012; revised 5 June 2012; accepted 11 June 2012; published online 6 September 2012 


\section{METHODS}

Study population

A total of 345 patients was consecutively recruited at the Hypertension Unit of the Division of Cardiology at Sant'Andrea Hospital in Rome. Patients underwent a full medical history, standard physical examination, blood pressure measurement and standard 12-lead electrocardiography. Clinical blood pressure was measured by a qualified nurse with a mercury sphygmomanometer and was estimated as the average of at least three measurements. Hypertension was diagnosed according to the European guidelines. ${ }^{9}$ Body weight and height were measured in light clothing without shoes, and body mass index (BMI) was calculated for all patients. Waist circumference was also measured while standing using a soft measuring tape, midway between the lowest rib and the iliac crest. MetS was defined using the ATP III criteria, ${ }^{10}$ and patients were classified as having MetS if they had at least three or more of the following risk factors: (1) waist circumference $>102 \mathrm{~cm}$ for men and $>88 \mathrm{~cm}$ for women; (2) triglycerides $\geqslant 150 \mathrm{mg} \mathrm{dl}^{-1}$; (3) high-density lipoprotein $<40 \mathrm{mg} \mathrm{dl}^{-1}$ in men or $<50 \mathrm{mg} \mathrm{dl}^{-1}$ in women; (4) systolic blood pressure $\geqslant 130 \mathrm{~mm} \mathrm{Hg}$, diastolic blood pressure $\geqslant 85 \mathrm{~mm} \mathrm{Hg}$ or undergoing hypertensive drug treatment in a patient with a history of hypertension; and (5) fasting glucose $\geqslant 100 \mathrm{mg} \mathrm{dl}^{-1}$ or use of hypoglycemic drugs. Diagnosis of diabetes was confirmed by fasting plasma glucose $>126 \mathrm{mg} \mathrm{dl}^{-1}$ in two separate determinations. Patients with previously known cardiovascular disease, peripheral arterial disease, chronic coronary artery disease, stable angina, positive stress testing or previous evidence of left or RV dysfunction were excluded from the study. Patients with rhythm disorders (atrial fibrillation and ventricular arrhythmias), valvular disease, or evidence of myocardial ischemia, familial dyslipidemia, dysthyroidism, liver or renal disease, malignancies, acute or chronic inflammatory conditions, chronic obstructive pulmonary disease or other causes of pulmonary hypertension were also excluded. Medical history was recorded directly from the patients. Routine biochemical measurements including fasting glucose, lipid profile and high-sensitivity C-reactive protein (hs-CRP) were performed. The study was approved by our Institute Committee, and all patients signed an informed consent form before entering the study. According to the ATP III criteria and the presence of DM II, the study population was divided in four groups: (1) healthy controls $(n=120)$; (2) MetS without T2DM ( $n=84)$; (3) T2DM without MetS ( $n=49)$; and (4) MetS plus T2DM $(n=92)$.

\section{Echocardiography}

All patients underwent trans-thoracic echocardiography, including both conventional and tissue Doppler imaging (TDI) of the LV and the RV. Echocardiography included two-dimensional and M-mode evaluation (Acuson Sequoia C 256). Doppler-echocardiographical exams were performed with a phased array sector scan, using a mutifrequency probe at 2.5, 3.5 or $5 \mathrm{MHz}$. Exams were performed by two different skilled sonographers (GT and GMC). Intraclass correlation coefficient and coefficient of variation were used to test the intra- and interobserver reliability of echocardiographical measurements. The following interpretation was used for intraclass correlation coefficient: poor $(<0.20)$, fair $(0.21-0.40)$, satisfactory $(0.41-0.60)$, good $(0.61-0.80)$ and very good $(0.81-1.00)$. Variability was classified as follows: very low $(<5 \%)$, low (5-15\%), moderate $(15-25 \%)$ and high $(>25 \%)$. Intra- and inter-observer reproducibility results were satisfactory for all measurements. TDI measurements in particular exhibited good reliability (intraclass correlation coefficient: $0.84,0.79$ and 0.87 ; coefficient of variation (\%): 11.5, 14.1 and 9.0 for lateral tricuspid, septal tricuspid and mitral annulus, respectively). LV diameters and wall thickness were measured according to the American Society of Echocardiography. ${ }^{11}$ Fractional shortening (FS) was calculated as ((LVEDDLVESD)/LVEDD) $\times 100 . \mathrm{LV}$ volumes and ejection fraction $(\mathrm{EF})$ were estimated using Simpson's technique in the four-chamber view. ${ }^{12} \mathrm{LV}$ mass was calculated using Devereux's formula and normalized by height. ${ }^{2,7}$ Pulsed-wave Doppler of mitral inflow velocity was performed as described previously. ${ }^{13,14}$ TDI of both ventricles was also performed. Sm (peak myocardial systolic velocity), Em (early diastolic myocardial velocity) and Am (late peak diastolic myocardial velocity) were measured. The $\mathrm{E} / \mathrm{Em}$ ratio, an index of ventricular filling pressure, was calculated. ${ }^{15,16}$ Ejection time (ET), isovolumic relaxation time (IVRT) and isovolumic contraction time were also derived. The myocardial performance index (MPI) of the LV was calculated as described previously. ${ }^{15}$ RV diameters were measured in the long axis view. ${ }^{15}$ The RV EF was calculated from the apical-four chamber view using Simpson's formula. ${ }^{15-17}$ RV early (E) and late (A) ventricular inflow velocities were measured by pulse-wave Doppler, placing the sample volume between the tips of the tricuspid valve in the apical four-chamber view. ${ }^{15}$ The maximal tricuspid regurgitation velocity was measured by continuous wave Doppler echocardiography in the four-chamber view, and Bernoulli's modified equation was used to calculate the systolic pulmonary pressure. To better estimate RV function, TDI was used with a multi-segmental approach. Regional pulsed TDI data were obtained from the lateral and septal tricuspid annulus in the apical four-chamber view. ${ }^{15-18}$ TDI MPI of the RV (RV MPI) was calculated as the sum of isovolumic relaxation and contraction times divided by ET ((IVRT + IVRT)/ ET), as described previously. ${ }^{15}$ The average RV MPI was assessed using the following formula: (MPI lateral $\left.+\mathrm{MPI}_{\text {septal }}\right) / 2$. RV and $\mathrm{LV}$ dysfunction was identified as MPI values $>2$ s.d. from the mean of the values derived from a group of 120 healthy subjects (TDI-derived MPI values $>0.56$ and 0.60 defined the presence of RV and LV dysfunction, respectively).

\section{Statistical analysis}

The data were analyzed using standard statistical software (version 13.0, SPSS, Chicago, IL, USA). Numerical data were reported as the mean \pm s.d., and categorical variables were expressed as percentage values (\%). Analysis of variance was used to assess linear trends of parameters in the four groups, and post-hoc testing was undertaken using the Bonferroni post-hoc test. Categorical variables were compared using a $\chi^{2}$ analysis. The between-variable correlations were measured by Pearson's correlation. Linear and logistic regression models, adjusted for confounding factors, were performed to test the association between indices of biventricular function and the presence of MetS, T2DM or their association. A two-tailed $P$-value $<0.05$ was considered the threshold to declare significance.

\section{RESULTS}

\section{Characteristics of the study sample}

The main anthropometric, clinical and biochemical features of the study population, stratified according to the presence of T2DM and MetS, are reported in Table 1. The groups did not exhibit any significant difference with respect to age, gender, heart rate, smoking habit or cardiovascular medications (Table 1). As expected, patients with MetS, T2DM or both had a higher BMI, waist circumference, systolic blood pressure and diastolic blood pressure as well as fasting glucose, cholesterol, triglyceride and hs-CRP levels when compared with their age-matched healthy controls (Table 1). The duration of the disease did not differ among diabetics with and without MetS. BMI, waist circumference and high-density lipoprotein cholesterol were significantly different in patients with MetS and T2DM compared with controls (Table 1). Fasting glucose and hs-CRP were significantly higher in the MetS and T2DM groups than in control subjects, and a further increase was observed in patients with both conditions.

\section{LV geometry and function}

Patients with MetS, T2DM or both exhibited a significantly higher LV diameter, wall thickness, LV mass and relative wall thickness compared with healthy controls (Table 2). Similarly, the indices of LV systolic and diastolic function were strongly impaired in the three groups. However, patients with T2DM and MetS did not significantly differ with respect to indices of LV geometry and function. Interestingly, geometric alterations and derangement of LV systo-diastolic indices were further enhanced in patients having both MetS and T2DM. Indeed, this latter group exhibited an increased prevalence of concentric geometry, higher LV mass, reduced EF, FS and peak myocardial systolic velocity velocity and a higher MPI, suggesting an impairment of both systolic and diastolic properties of 
Table 1 Anthropometric, clinical and biochemical characteristics of the study population

\begin{tabular}{|c|c|c|c|c|c|}
\hline & Controls $(n=120)$ & MetS $(\mathrm{n}=84)$ & $T 2 D M(\mathrm{n}=49)$ & $M e t S+T 2 D M(n=92)$ & $\mathrm{P}$-value \\
\hline Age & $51.8 \pm 10$ & $53.3 \pm 9.9$ & $53.7 \pm 12$ & $55.0 \pm 9.9$ & NS \\
\hline Gender ( $\mathrm{M}: \mathrm{F})$ & $80: 40$ & $57: 27$ & $16: 33$ & $34: 58$ & NS \\
\hline $\mathrm{BMI}\left(\mathrm{kg} \mathrm{m}^{-2}\right)$ & $22.8 \pm 1.4$ & $26.6 \pm 4.3$ & $26.3 \pm 4.8$ & $28.9 \pm 4.6$ & $<0.001$ \\
\hline Waist circumference (cm) & $86.6 \pm 8.8$ & $101.5 \pm 9.0$ & $97.2 \pm 9.1$ & $106.9 \pm 12$ & $<0.001$ \\
\hline HR (beats $\min ^{-1}$ ) & $68.3 \pm 10$ & $70.3 \pm 12.4$ & $71.1 \pm 12.8$ & $68.6 \pm 13$ & NS \\
\hline $\mathrm{SBP}(\mathrm{mm} \mathrm{Hg})$ & $121.7 \pm 11$ & $143.0 \pm 14.6$ & $137.7 \pm 16.2$ & $144.5 \pm 17$ & $<0.001$ \\
\hline $\mathrm{DBP}(\mathrm{mm} \mathrm{Hg})$ & $75.1 \pm 8.6$ & $90.4 \pm 8.1$ & $84.2 \pm 9.7$ & $88.66 \pm 11$ & $<0.001$ \\
\hline Current smokers (\%) & 30.83 & 21.4 & 36.7 & 34.7 & NS \\
\hline Duration of diabetes (years) & $-1-$ & $-1-$ & $4.1 \pm 2.4$ & $4.6 \pm 3.8$ & NS \\
\hline Glucose $\left(\mathrm{mgdl}{ }^{-1}\right)$ & $79.1 \pm 11$ & $91.9 \pm 9.2$ & $111.8 \pm 26.0$ & $117.8 \pm 21$ & $<0.001$ \\
\hline Total cholesterol $\left(\mathrm{mg} \mathrm{dl}^{-1}\right)$ & $174.3 \pm 25$ & $192.0 \pm 31.4$ & $194.7 \pm 36.1$ & $200.4 \pm 28$ & $<0.001$ \\
\hline HDL cholesterol (mg dl $\left.{ }^{-1}\right)$ & $60.5 \pm 9.0$ & $48.4 \pm 16.9$ & $59.5 \pm 20.9$ & $45.3 \pm 8.1$ & $<0.001$ \\
\hline LDL cholesterol $\left(\mathrm{mg} \mathrm{dl}^{-1}\right)$ & $91.9 \pm 28$ & $112.4 \pm 31.2$ & $112.6 \pm 35.9$ & $124.7 \pm 29$ & $<0.001$ \\
\hline Triglycerides $\left(\mathrm{mg} \mathrm{dl}^{-1}\right)$ & $109.1 \pm 22$ & $155.1 \pm 49.6$ & $112.8 \pm 44.5$ & $151.8 \pm 63$ & $<0.001$ \\
\hline hs-CRP (mg dl-1) & $0.16 \pm 0.1$ & $0.45 \pm 0.2$ & $0.47 \pm 0.2$ & $0.57 \pm 0.3$ & $<0.001$ \\
\hline ACE-I (\%) & $-1-$ & 42.8 & 48.9 & 57.6 & NS \\
\hline ARBs (\%) & $-1-$ & 36.9 & 34.7 & 30.4 & NS \\
\hline$\beta$-Blockers (\%) & $-1-$ & 20.2 & 24.4 & 26.0 & NS \\
\hline Ca-antagonists (\%) & $-1-$ & 27.3 & 22.4 & 27.1 & NS \\
\hline Diuretics (\%) & $-1-$ & 20.2 & 26.5 & 26.0 & NS \\
\hline Lipid lowering agents (\%) & $-1-$ & 51.5 & 48.9 & 59.7 & NS \\
\hline
\end{tabular}

Abbreviations: ACE, angiotensin converting enzyme; ANOVA, analysis of variance; ARBs, angiotensin II receptor blockers; BMI, body mass index; DBP, diastolic blood pressure; F, female; HDL, high-density lipoprotein; HR, heart rate; hs-CRP, high-sensitivity C-reactive protein; LDL, low-density lipoprotein; M, male; MetS, metabolic syndrome; NS, not significant; SBP, systolic blood pressure; T2DM, type 2 diabetes.

Values are expressed as mean \pm s.d.

$P$-value refers to ANOVA and $\chi^{2}$-tests.

Table 2 Conventional and TDI-derived echocardiografic parameters of the LV across the four groups

\begin{tabular}{|c|c|c|c|c|c|}
\hline & Controls $(n=120)$ & MetS $(n=84)$ & $T 2 D M(n=49)$ & $M e t S+T 2 D M(\mathrm{~N}=92)$ & P-value \\
\hline LVEDD (mm) & $47.38 \pm 5.42$ & $51.55 \pm 4.06$ & $50.33 \pm 3.24$ & $53.79 \pm 3.73$ & $<0.001$ \\
\hline LVESD (mm) & $27.61 \pm 4.13$ & $31.26 \pm 5.29$ & $31.33 \pm 3.56$ & $35.10 \pm 4.05$ & $<0.001$ \\
\hline IVSTd (mm) & $9.04 \pm 1.64$ & $10.16 \pm 1.40$ & $10.29 \pm 1.36$ & $11.85 \pm 1.51$ & $<0.001$ \\
\hline PWTd (mm) & $8.87 \pm 1.51$ & $10.50 \pm 1.04$ & $10.24 \pm 0.87$ & $11.49 \pm 1.07$ & $<0.001$ \\
\hline LV mass (g) & $152.48 \pm 51.63$ & $205.67 \pm 39.69$ & $195.16 \pm 32.51$ & $258.93 \pm 43.83$ & $<0.001$ \\
\hline LV mass ${ }^{2.7}\left(\mathrm{~g} \mathrm{~m}^{-2.7}\right)$ & $35.87 \pm 11.64$ & $48.89 \pm 9.51$ & $47.22 \pm 9.01$ & $63.48 \pm 14.63$ & $<0.001$ \\
\hline RWT & $0.37 \pm 0.05$ & $0.40 \pm 0.04$ & $0.40 \pm 0.04$ & $0.43 \pm 0.04$ & $<0.001$ \\
\hline $\mathrm{EF}(\%)$ & $66.55 \pm 8.03$ & $63.65 \pm 10.27$ & $62.15 \pm 6.20$ & $57.94 \pm 8.51$ & 0.01 \\
\hline FS (\%) & $41.60 \pm 6.83$ & $39.43 \pm 8.31$ & $37.84 \pm 5.06$ & $34.73 \pm 6.35$ & $<0.05$ \\
\hline E/A ratio & $1.20 \pm 0.14$ & $1.02 \pm 0.38$ & $0.92 \pm 0.26$ & $0.79 \pm 0.15$ & $<0.001$ \\
\hline $\mathrm{DT}(\mathrm{ms})$ & $175.71 \pm 57.58$ & $203.41 \pm 49.14$ & $207.38 \pm 39.91$ & $232.80 \pm 43.79$ & 0.03 \\
\hline $\mathrm{Em}\left(\mathrm{ms}^{-1}\right)$ & $0.214 \pm 0.061$ & $0.161 \pm 0.035$ & $0.168 \pm 0.046$ & $0.125 \pm 0.030$ & $<0.001$ \\
\hline $\mathrm{Am}\left(\mathrm{ms}^{-1}\right)$ & $0.158 \pm 0.051$ & $0.175 \pm 0.050$ & $0.191 \pm 0.045$ & $0.198 \pm 0.053$ & $<0.001$ \\
\hline IVRT (ms) & $70.48 \pm 18.77$ & $82.05 \pm 19.67$ & $83.06 \pm 19.93$ & $92.21 \pm 19.99$ & $<0.001$ \\
\hline $\mathrm{Em} / \mathrm{Am}$ & $1.49 \pm 0.69$ & $0.99 \pm 0.33$ & $0.94 \pm 0.38$ & $0.67 \pm 0.21$ & $<0.001$ \\
\hline $\mathrm{E} / \mathrm{Em}$ & $3.37 \pm 1.39$ & $4.23 \pm 1.62$ & $4.01 \pm 1.20$ & $6.00 \pm 1.71$ & $<0.001$ \\
\hline IVCT (ms) & $62.92 \pm 11.22$ & $67.55 \pm 12.50$ & $69.27 \pm 15.58$ & $73.27 \pm 14.98$ & $<0.001$ \\
\hline ET (ms) & $294.71 \pm 32.59$ & $284.19 \pm 34.38$ & $298.22 \pm 34.37$ & $275.07 \pm 29.87$ & $<0.001$ \\
\hline $\mathrm{Sm}\left(\mathrm{ms}^{-1}\right)$ & $0.183 \pm 0.036$ & $0.149 \pm 0.032$ & $0.153 \pm 0.035$ & $0.129 \pm 0.029$ & $<0.001$ \\
\hline LV MPI & $0.46 \pm 0.09$ & $0.53 \pm 0.09$ & $0.51 \pm 0.11$ & $0.61 \pm 0.10$ & $<0.001$ \\
\hline
\end{tabular}

Abbreviations: Am, late diastolic myocardial velocity; ANOVA, analysis of variance; DT, deceleration time; EF, ejection fraction; Em, early diastolic myocardial velocity; ET, ejection time; FS, fractional shortening; IVCT, isovolumic contraction time; IVRT, isovolumic relaxation time; IVSTd, interventricular septum thickness diastole; LV, left ventricular; LVEDD, LV end diastolic diameter; LVESD, LV end systolic diameter; LVM, LV mass; LV MPI, LV myocardial performance index; PWTd, posterior wall thickness diastole; RWT, relative wall thickness; Sm, peak myocardial systolic velocity; TDI, tissue Doppler imaging; T2DM, type 2 diabetes.

Values are expressed as mean $\pm \mathrm{s}$.d.

$P$-value refers to ANOVA test. 


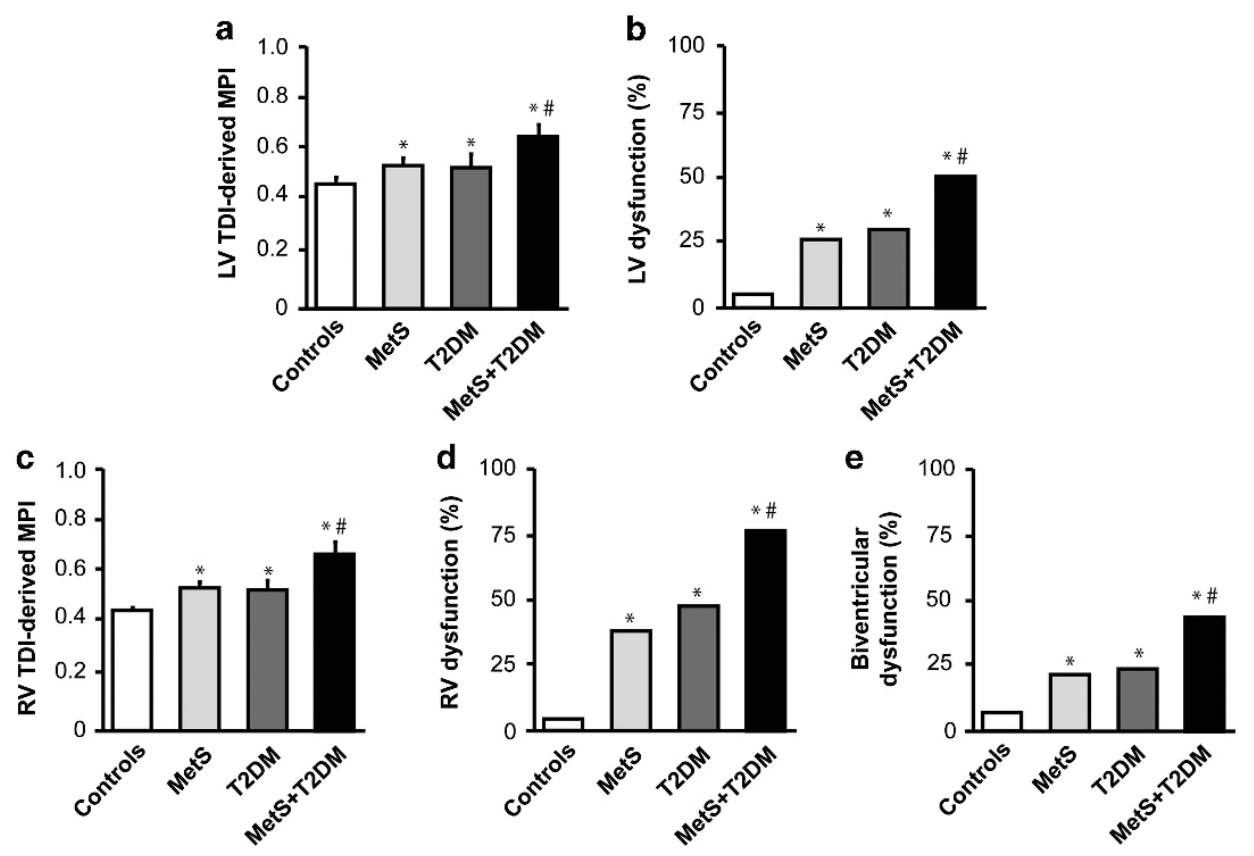

Figure 1 Preclinical effect of MetS, T2DM and their association on biventricular function. (a and $\mathbf{b}$ ) LV MPI values and prevalence of LV dysfunction; (c and d) RV MPI values and prevalence of RV dysfunction. (e) Prevalence of biventricular dysfunction across the four groups. Values are expressed as mean \pm s.d. (a and $\mathbf{c}$ ) and percentages (b, $\mathbf{d}$ and $\mathbf{e})$. ANOVA and $\chi^{2}$-test were used where appropriate. ${ }^{*} P<0.05$ vs. controls; ${ }^{*} P<0.05$ vs. controls, MetS and T2DM. ANOVA, analysis of variance; LV, left ventricle; MetS, metabolic syndrome; T2DM, type 2 diabetes, MPI, myocardial performance index; RV, right ventricle.

the LV (Figure 1a). Diastolic dysfunction was also confirmed by a significant increase in the deceleration time (DT), IVRT and E/Em ratio (Table 2). The prevalence of LV dysfunction, identified by MPI, was similar in the MetS and T2DM groups but significantly increased in the presence of both conditions ( $26 \% v$ s. $28 \% v$ s. $52 \%$, respectively, Figure 1b).

\section{Indices of RV function}

Table 3 presents the indices of RV function across the four groups. Patients with MetS, T2DM or their association exhibited significantly higher RV diameters, increased volumes and a consistent impairment of systo-diastolic function compared with healthy controls (Table 3). $\mathrm{RV}$ size and function were comparable between MetS and T2DM groups, but a further decline was observed in patients with both conditions. MPI-derived RV dysfunction was higher in the MetS and DM groups compared with controls $(37 \%$ vs. $47 \%$ and $3 \%$, respectively). Interestingly, the association of MetS and T2DM resulted in a further impairment of RV function, as assessed by TDI-derived MPI (77\%, $P<0.01$ vs. MetS and T2DM, Figures 1c and $\mathrm{d})$. With respect to systolic function, this latter group exhibited a substantial reduction of EF, FS, peak myocardial systolic velocity and a significant increase in the RV MPI (Figure 1c). A progressive impairment of diastolic function was also observed across the four groups, as suggested by a significant reduction of Em velocity and the Em/Am ratio, as well as by a consistent increase in IVRT and the E/Em ratio (Table 3).

\section{MetS components, inflammation and risk of biventricular} dysfunction

Systolic blood pressure $(r=0.32, r=0.27)$, diastolic blood pressure $(r=0.25, r=0.28)$, high-density lipoprotein cholesterol $(r=-0.28$, $r=-0.21)$, fasting glucose $(r=0.33, r=0.37)$ and waist circumference $(r=0.22, r=0.28)$ significantly correlated with both RV and LV
MPI, respectively. Interestingly, the hs-CRP increase paralleled the MPI value of both ventricles $(r=0.38, r=0.32)$. Logistic regression models adjusted for age, gender, BMI and LV mass ${ }^{2,7}$ indicated that individual MetS components were independently associated with RV and LV dysfunction. However, the association between MetS and biventricular dysfunction was stronger than for individual components and was comparable to T2DM (Table 4). Interestingly, patients with both MetS and T2DM exhibited the highest risk of biventricular dysfunction compared with MetS or T2DM (47\% vs. $25 \%$ vs. $23 \%$, respectively, Table 4, Figure 1e). Interestingly, hs-CRP emerged as a strong independent predictor of RV and LV dysfunction when compared with traditional cardiovascular risk factors (Table 4).

\section{DISCUSSION}

In this study, we have observed that patients with MetS and T2DM exhibit a similar impairment of biventricular function compared with age-matched healthy controls. Moreover, we observed a synergic effect between these two clinical conditions; the largest decline of RV and LV function was observed in patients having both MetS and T2DM.

Previous studies have explored the preclinical effects of MetS and T2DM on cardiac function. ${ }^{19-23}$ It has been recently reported that patients with T2DM have an increased prevalence of biventricular dysfunction as a result of myocardial steatosis. In this study, myocardial triglyceride content was an independent correlate of biventricular longitudinal strain. ${ }^{20}$ However, recent investigations have shown that MetS associates with diastolic dysfunction and subtle impairment of systolic properties. ${ }^{5,22,23}$ Indeed, an increasing body of evidence supports the notion that a constellation of risk factors, as observed in MetS, may resemble the pathological features of diabetic cardiomyopathy. ${ }^{21-23}$ However, the data for a direct comparison between MetS and T2DM, as well as the impact of their association on cardiac geometry and function, are not exhaustive. This issue needs to be clarified because the long-term 
Table 3 Conventional and TDI-derived echocardiografic parameters of the RV across the four groups

\begin{tabular}{|c|c|c|c|c|c|}
\hline & Controls $(n=120)$ & MetS $(\mathrm{N}=84)$ & $T 2 D M(n=49)$ & MetS + T2DM $(\mathrm{N}=92)$ & P-value \\
\hline RVEDD (mm) & $16.65 \pm 2.55$ & $21.98 \pm 3.87$ & $21.67 \pm 3.91$ & $23.42 \pm 3.25$ & $<0.001$ \\
\hline RVESD (mm) & $10.59 \pm 1.77$ & $14.84 \pm 2.32$ & $14.77 \pm 2.36$ & $16.74 \pm 1.97$ & $<0.001$ \\
\hline RVESV (ml) & $2.55 \pm 1.18$ & $6.18 \pm 2.52$ & $6.12 \pm 2.76$ & $8.27 \pm 2.61$ & $<0.001$ \\
\hline RVEDV/BSA $\left(\mathrm{ml} \mathrm{m}^{-2}\right)$ & $4.71 \pm 1.96$ & $9.03 \pm 3.96$ & $8.89 \pm 4.24$ & $10.14 \pm 3.47$ & $<0.001$ \\
\hline$E F(\%)$ & $69.32 \pm 6.40$ & $62.17 \pm 8.95$ & $61.55 \pm 7.97$ & $56.55 \pm 7.95$ & $<0.001$ \\
\hline \multicolumn{6}{|l|}{ Lateral tricuspid TDI } \\
\hline $\mathrm{Em}\left(\mathrm{ms}^{-1}\right)$ & $0.209 \pm 0.039$ & $0.165 \pm 0.033$ & $0.174 \pm 0.030$ & $0.141 \pm 0.033$ & $<0.001$ \\
\hline$A m\left(m s^{-1}\right)$ & $0.154 \pm 0.039$ & $0.200 \pm 0.045$ & $0.203 \pm 0.043$ & $0.219 \pm 0.041$ & $<0.001$ \\
\hline IVRT (ms) & $64.57 \pm 15.38$ & $82.11 \pm 14.11$ & $86.94 \pm 19.30$ & $96.29 \pm 14.18$ & $<0.001$ \\
\hline $\mathrm{Em} / \mathrm{Am}$ & $1.44 \pm 0.49$ & $0.85 \pm 0.19$ & $0.87 \pm 0.16$ & $0.66 \pm 0.18$ & $<0.001$ \\
\hline $\mathrm{E} / \mathrm{Em}$ & $3.85 \pm 1.14$ & $4.31 \pm 1.21$ & $4.71 \pm 1.64$ & $6.87 \pm 4.28$ & $<0.001$ \\
\hline \multicolumn{6}{|l|}{ Septal tricuspid TDI } \\
\hline $\mathrm{Em}\left(\mathrm{ms}^{-1}\right)$ & $0.161 \pm 0.040$ & $0.140 \pm 0.026$ & $0.146 \pm 0.026$ & $0.117 \pm 0.024$ & $<0.001$ \\
\hline$A m\left(m^{-1}\right)$ & $0.124 \pm 0.036$ & $0.149 \pm 0.032$ & $0.160 \pm 0.032$ & $0.163 \pm 0.032$ & $<0.001$ \\
\hline IVRT (ms) & $68.53 \pm 15.94$ & $88.05 \pm 17.68$ & $86.69 \pm 16.54$ & $100.73 \pm 19.41$ & $<0.001$ \\
\hline $\mathrm{Em} / \mathrm{Am}$ & $1.48 \pm 1.09$ & $0.96 \pm 0.22$ & $0.94 \pm 0.23$ & $0.74 \pm 0.19$ & $<0.001$ \\
\hline $\mathrm{E} / \mathrm{Em}$ & $4.97 \pm 1.63$ & $4.98 \pm 1.56$ & $4.84 \pm 1.13$ & $5.25 \pm 1.84$ & 0.45 \\
\hline IVCT (ms) & $67.72 \pm 12.83$ & $67.18 \pm 13.05$ & $66.59 \pm 15.90$ & $73.14 \pm 16.27$ & $<0.001$ \\
\hline ET (ms) & $292.59 \pm 30.87$ & $287.76 \pm 31.84$ & $295.67 \pm 36.17$ & $272.91 \pm 35.75$ & $<0.001$ \\
\hline $\mathrm{Sm}\left(\mathrm{ms}^{-1}\right)$ & $0.159 \pm 0.055$ & $0.140 \pm 0.025$ & $0.143 \pm 0.020$ & $0.117 \pm 0.022$ & $<0.001$ \\
\hline Septal MPI & $0.45 \pm 0.07$ & $0.54 \pm 0.08$ & $0.52 \pm 0.08$ & $0.65 \pm 0.15$ & $<0.001$ \\
\hline Average TDI MPI & $0.44 \pm 0.05$ & $0.53 \pm 0.04$ & $0.53 \pm 0.06$ & $0.65 \pm 0.10$ & $<0.001$ \\
\hline
\end{tabular}

Abbreviations: Am, late diastolic myocardial velocity; ANOVA, analysis of variance; BSA, body surface area; EF, ejection fraction; Em, early diastolic myocardial velocity; ET, ejection time; FS, fractional shortening; IVCT, isovolumic contraction time; IVRT, isovolumic relaxation time; MPI, myocardial performance index; RVEDD, right ventricular end diastolic diameter; RVEDV, RV end diastolic volume; RVESD, RV end systolic diameter; RVESV, RV end systolic volume; Sm, peak myocardial systolic velocity; TDI, tissue Doppler imaging; T2DM, type 2 diabetes.

Values are expressed as mean \pm s.d.

$P$-value refers to ANOVA test.

Table 4 Logistic regression analysis adjusted for age, gender, BMI and $\mathrm{LVM}^{2,7}$ showing the risk of biventricular dysfunction of individual MetS components, MetS, T2DM and their association

\begin{tabular}{lcc}
\hline & \multicolumn{2}{c}{ Biventricular dysfunction } \\
\cline { 2 - 3 } Variable & OR (Cl 95\%) & P-value \\
\hline SBP & $1.03(0.98-1.05)$ & 0.13 \\
DBP & $1.07(0.97-1.11)$ & 0.12 \\
Triglycerides & $1.14(1.02-3.82)$ & $<0.05$ \\
HDL cholesterol & $0.97(0.95-0.99)$ & $<0.05$ \\
Fasting glycemia & $1.10(1.07-4.04)$ & $<0.05$ \\
Waist circumference & $1.22(1.07-3.91)$ & $<0.05$ \\
Hs-CRP & $2.35(1.06-6.97)$ & $<0.05$ \\
MetS & $3.90(1.6-9.4)$ & $<0.001$ \\
T2DM & $3.77(1.9-13.4)$ & $<0.01$ \\
MetS + T2DM & $5.5(2.5-12.4)$ & $<0.001$ \\
\hline
\end{tabular}

Abbreviations: BMI, body mass index; $\mathrm{Cl}$, confidence interval; DBP, diastolic blood pressure; HDL, high-density lipoprotein; Hs-CRP, high-sensitivity $\mathrm{C}$ reactive protein; LVM, left ventricular mass; MetS, metabolic syndrome; MPI, myocardial performance index; OR, odds ratio; RV, right mass; MetS, metabolic syndrome; MPI, myocardial performance index; OR, odds ratio; RV, right
ventricular; SBP, systolic blood pressure; TDI, tissue Doppler imaging; T2DM, type 2 diabetes. ventricular; SBP, systolic blood pressure; TDI, tissue Doppler imaging; T2DM, typ
Biventricular dysfunction was defined basing on TDI-derived MPI of LV and RV. risk of HF in patients with MetS has been reported to be largely inferior to the risk of diabetic patients. ${ }^{8}$ Indeed, individual risk factors rather than MetS 'per se' were associated with incident HF over time. ${ }^{8}$ European guidelines on the management of arterial hypertension classify MetS as the equivalent of T2DM, without considering their coexistence in a different risk category. ${ }^{9}$ This issue deserves attention because a large number of diabetic patients present at diagnosis with MetS criteria, and the appropriate management of these patients is not well defined. Such uncertainty prompted us to design a study aimed at investigating the prevalence of preclinical cardiac organ damage in patients with MetS, T2DM or their association. All patients enrolled were free of cardiovascular disease and had a preserved LV EF. These criteria allowed us to better investigate the relationship between metabolic abnormalities and the systo-diastolic properties of both ventricles. Moreover, biventricular function was assessed with TDI-derived MPI, which is emerging as a reliable tool for the detection of subtle abnormalities of cardiac performance. ${ }^{13-17,24}$ In our study, the MPI of the RV was derived with a multisegmental approach that took into consideration the myocardial velocities measured both at the RV lateral wall and at the interventricular septum. Owing to RV geometry, functional data obtained with TDI 
provide a more reliable estimation of chamber function compared with traditional indices such as EF. Indeed, TDI evaluation of the RV strongly correlates with cardiac magnetic resonance estimations. ${ }^{18}$ More importantly, MPI offers a simultaneous evaluation of systolic and diastolic function because in its formula, isovolumic relaxation and contraction times, as well as the ventricular ET, are included. ${ }^{15,25}$ In our study, MPI values $>0.56$ (RV) and $>0.60$ (LV) were considered abnormal according to variable distribution in a control group of healthy subjects. Although our study did not assess the prognostic impact of such MPI cutoffs, similar values were powerful predictors of cardiovascular mortality in patients with preserved systolic function. ${ }^{26}$ By contrast, mitral inflow-derived MPI exhibited a poor prognostic value in the population of the Strong Heart Study. ${ }^{27}$ A larger prevalence of obesity, hypertension and diabetes in our study as well as a different gender and ethnicity distribution may contribute to explain the better MPI performance that emerged from our analysis. Notably, in our study, MPI was derived from TDI measurements with myocardial longitudinal velocities averaged to obtain an accurate estimation of isovolumic contraction time, IVRT and ET. Hence, TDI-MPI is emerging as a reliable and attractive tool for the evaluation of biventricular function, particularly at the preclinical stage. $^{28,29}$

In this study, we provide an exhaustive echocardiographical evaluation comparing patients with MetS alone, those with T2DM in the absence of MetS criteria and patients with both MetS and T2DM. We also determined that MetS was a stronger predictor of deteriorating biventricular function than individual components. Indeed, adjusted odds ratios of waist circumference, high-density lipoprotein cholesterol, triglycerides and blood pressure were all inferior when compared with their clustering, as observed in MetS.

Alterations of cardiac geometry and function in the setting of MetS and T2DM may be the result of insulin resistance and hyperglycemia. Both of these conditions activate pathways responsible for cardiac hypertrophy, myocardial fibrosis and perivascular inflammation..$^{5,30-32}$ In this study, we found that MetS, a condition of insulin resistance in the absence of hyperglycemia, has the same impact as T2DM on cardiac geometry and function. Interestingly, when considering patients with both MetS and T2DM, we observed further impairment of LV and RV function, suggesting a synergic effect of these two clinical entities on cardiac organ damage.

Recent evidence suggests that systemic inflammation may have a pivotal role as a strong risk predictor for incident $\mathrm{HF}^{8}$ In particular, hs-CRP is emerging as a nontraditional risk factor in the setting of cardiometabolic disorders. ${ }^{5,8}$ However, the usefulness of such an inflammatory index in the prediction of cardiac organ damage in the absence of traditional risk factors is not well defined. In this study, hs-CRP was similarly increased in both MetS and T2DM patients compared with controls. Interestingly, hs-CRP levels further rose when considering patients with the combination of MetS and T2DM, suggesting an additive effect of insulin resistance and hyperglycemia on systemic inflammation. Notably, hs-CRP positively correlated with the MPI of both ventricles and was a predictor of biventricular dysfunction regardless of age, gender, BMI and LV mass. Moreover, individual MetS components were all inferior to hs-CRP in predicting cardiac dysfunction. This latter finding supports the superiority of hs-CRP compared with individual MetS components in our setting (Table 4). In line with this interpretation, a recent study reported that hs-CRP has an important role in the association between obesity and HF development. ${ }^{8} \mathrm{~A}$ limitation of this study is represented by the lack of data on incident HF supporting our assumptions. However, prospective studies support the idea that MPI is a reliable predictor of
HF and cardiovascular outcomes. ${ }^{28}$ Our study set the stage for larger investigations tailored to address the long-term risk of HF associated with MetS, T2DM and their association.

In conclusion, we report that MetS and T2DM equally affect biventricular function, and their association contributes to further detrimental function. Our findings suggest that the functional aspects of diabetic cardiomyopathy are already manifest in patients with MetS. Individuals with both MetS and T2DM should be considered to be in a higher risk category than MetS or T2DM alone and should receive an earlier and more aggressive pharmacological treatment to delay the progression of chamber dysfunction toward HF.

\section{CONFLICT OF INTEREST}

The authors declare no conflict of interest.

1 Nichols GA, Gullion CM, Koro CE, Ephross SA, Brown JB. The incidence of congestive heart failure in type 2 diabetes: an update. Diabetes Care 2004; 27: 1879-1884.

2 Bertoni AG, Hundley WG, Massing MW, Bonds DE, Burke GL, Goff Jr DC. Heart failure prevalence, incidence, and mortality in the elderly with diabetes. Diabetes Care 2004; 27: 699-703.

3 Barac A, Panza J, Howard BV, de Simone G, Devereux RB, Chinali M, Lee ET, Galloway JM. Diabetes and Incident Heart Failure: the Strong Heart Study. High Blood Press Cardiovas Prev 2008; 15: 171-215.

4 Iribarren C, Karter AJ, Go AS, Ferrara A, Liu JY, Sidney S, Selby JV. Glycemic control and heart failure among adult patients with diabetes. Circulation 2001; 103: 2668-2673.

5 Sciarretta S, Ferrucci A, Ciavarella GM, De Paolis P, Venturelli V, Tocci G, De Biase L, Rubattu S, Volpe M. Markers of inflammation and fibrosis are related to cardiovascular damage in hypertensive patients with metabolic syndrome. Am J Hypertens 2007; 20: 784-791.

6 Butler J, Rodondi N, Zhu Y, Figaro K, Fazio S, Vaughan DE, Satterfield S, Newman AB, Goodpaster B, Bauer DC, Holvoet P, Harris TB, de Rekeneire N, Rubin S, Ding J, Kritchevsky SBHealth ABC Study. Metabolic syndrome and the risk of cardiovascular disease in older adults. J Am Coll Cardiol 2006; 47: 1595-1602.

7 de Simone G, Devereux RB, Chinali M, Lee ET, Howard B. Interaction of metabolic syndrome and left ventricular hypertrophy in the prediction of cardiovascular risk: the Strong Heart Study. High Blood Press Cardiovas Prev 2007; 14: 145-196.

8 Bahrami H, Bluemke DA, Kronmal R, Bertoni AG, Lloyd-Jones DM, Shahar E, Szklo M, Lima JA. Novel metabolic risk factors for incident heart failure and their relationship with obesity: the MESA (Multi-Ethnic Study of Atherosclerosis) study. J Am Coll Cardiol 2008; 51: 1775-1783.

9 Mancia G, De Backer G, Dominiczak A, Cifkova R, Fagard R, Germano G, Grassi G, Heagerty AM, Kjeldsen SE, Laurent S, Narkiewicz K, Ruilope L, Rynkiewicz A, Schmieder RE, Boudier HA, Zanchetti A, ESH-ESC Task Force on the Management of Arterial Hypertension. 2007 ESH-ESC Practice Guidelines for the Management of Arterial Hypertension: ESH-ESC Task Force on the Management of Arterial Hypertension. J Hypertens 2007; 25: 1751-1762.

10 Alberti KG, Eckel RH, Grundy SM, Zimmet PZ, Cleeman JI, Donato KA, Fruchart JC, James WP, Loria CM, Smith Jr SC. International diabetes federation task force on epidemiology and prevention. Circulation 2009; 120: 1640-1645.

11 Lang RM, Bierig M, Devereux RB, Flachskampf FA, Foster E, Pellikka PA, Picard MH, Roman MJ, Seward J, Shanewise JS, Solomon SD, Spencer KT, Sutton MS, Stewart WJ. Recommendations for chamber quantification: a report from the American Society of Echocardiography's Guidelines and Standards Committee and the Chamber Quantification Writing Group, developed in conjunction with the European Association of Echocardiography, a branch of the European Society of Cardiology. J Am Soc Echocardiogr 2005; 18: 1440-1463.

12 de Simone G, Devereux RB, Ganau A, Hahn RT, Saba PS, Mureddu GF, Roman MJ, Howard BV. Estimation of left ventricular chamber and stroke volume by limited M-mode echocardiography and validation by two-dimensional and Doppler echocardiography. Am J Cardiol 1996; 78: 801-807.

13 Paneni F, Gregori M, Ciavarella GM, Sciarretta S, Tocci G, Palano F, Domenici A, Punzo G, De Biase L, Menè P, Volpe M. Impact of dialysis modality on the appropriateness of left ventricular mass in patients with end-stage renal disease. Int J Cardiol 2011; 149: 250-252.

14 Sciarretta S, Paneni F, Ciavarella GM, De Biase L, Palano F, Baldini R, Quarta G, Tocci G, Benedetto U, Ferrucci A, Rubattu S, de Simone G, Volpe M. Evaluation of systolic properties in hypertensive patients with different degrees of diastolic dysfunction and normal ejection fraction. Am J Hypertens 2009; 22: 437-443.

15 Paneni F, Gregori M, Ciavarella GM, Sciarretta S, De Biase L, Marino L, Tocci G, Principe $F$, Domenici $A$, Luciani R, Punzo G, Menè $P$, Volpe M. Right ventricular dysfunction in patients with end-stage renal disease. Am J Nephrol 2010; 32: 432-438.

16 Paneni F, Gregori M, Ciavarella GM, Sciarretta S, Palano F, Pignatelli G, Castello L, Domenici A, Punzo G, Tocci G, De Biase L, Menè P, Volpe M. Relation between right and 
left ventricular function in patients undergoing chronic dialysis. J Cardiovasc Med 2011 (e-pub ahead of print 20 December 2011; doi:10.2459/JCM.0b013e32834 eacf0).

17 Jenkins C, Chan J, Bricknell K, Strudwick M, Marwick TH. Reproducibility of right ventricular volumes and ejection fraction using real-time threedimensional echocardiography: comparison with cardiac MRI. Chest 2007; 131 1844-1851.

18 Sade LE, Gülmez O, Ozyer U, Ozgül E, Ağildere M, Müderrisoğlu H. Tissue Doppler study of the right ventricle with a multisegmental approach: comparison with cardiac magnetic resonance imaging. J Am Soc Echocardiogr 2009; 22: 361-368.

19 Poulsen MK, Henriksen JE, Dahl J, Johansen A, Gerke O, Vach W, Haghfelt T, Høilund-Carlsen PF, Beck-Nielsen H, Møller JE. Left ventricular diastolic function in type 2 diabetes mellitus: prevalence and association with myocardial and vascular disease. Circ Cardiovasc Imaging 2010; 3: 24-31.

20 Ng AC, Delgado V, Bertini M, van der Meer RW, Rijzewijk LJ, Hooi Ewe S, Siebelink HM, Smit JW, Diamant M, Romijn JA, de Roos A, Leung DY, Lamb HJ, Bax JJ. Myocardial steatosis and biventricular strain and strain rate imaging in patients with type 2 diabetes mellitus. Circulation 2010; 122: 2538-2544.

21 Fang ZY, Schull-Meade R, Downey M, Prins J, Marwick TH. Determinants of subclinica diabetic heart disease. Diabetologia 2005; 48: 394-402.

22 Gong HP, Tan HW, Fang NN, Song T, Li SH, Zhong M, Zhang W, Zhang Y. Impaired left ventricular systolic and diastolic function in patients with metabolic syndrome as assessed by strain and strain rate imaging. Diabetes Res Clin Pract 2009; 83: 300-307.

23 Voulgari C, Moyssakis i, Papazafiropoulou A, Perrea D, Kyriaki D, Katsilambros N, Tentolouris N. The impact of metabolic syndrome on left ventricular myocardial performance. Diabetes Metab Res Rev 2010; 26: 121-127.
24 Moller J, Poulsen S, Egstrup K. Effect of preload alternations on a new Dopple echocardiographic index of combined systolic and diastolic performance. J Am Soc Echocardiogr 1999; 12: 1065-1072.

25 Paneni F, Gregori M, Pignatelli G, Ciavarella GM, Punzo G, De Biase L, Tocci G, Menè $P$, Volpe $M$. Pathophysiology of biventricular dysfunction during hemodialysis: emerging concepts. Int J Cardiol 2012; 155: 478-479.

26 Kim H, Yoon HJ, Park HS, Cho YK, Nam CW, Hur SH, Kim YN, Kim KB. Usefulness of tissue Doppler imaging-myocardial performance index in the evaluation of diastolic dysfunction and heart failure with preserved ejection fraction. Clin Cardiol 2011; 34 494-499.

27 Mishra RK, Kizer JR, Palmieri V, Roman MJ, Galloway JM, Fabsitz RR, Lee ET, Best LG, Devereux RB. Utility of the myocardial performance index in a population with high prevalences of obesity, diabetes, and hypertension: the strong heart study. Echocardiography 2007; 24: 340-347.

28 Arnlöv J, Lind L, Andrén B, Risérus U, Berglund L, Lithell HA. Doppler-derived index of combined left ventricular systolic and diastolic function is an independent predictor of cardiovascular mortality in elderly men. Am Heart J 2005; 149: 902-147.

29 Mogelvang R, Sogaard P, Pedersen SA, Olsen NT, Marott JL, Schnohr P, Goetze JP, Jensen JS. Cardiac dysfunction assessed by echocardiographic tissue Doppler imaging is an independent predictor of mortality in the general population. Circulation 2009; 119: 2679-2685.

30 Poornima IG, Parikh P, Shannon RP. Diabetic cardiomyopathy: the search for a unifying hypothesis. Circ Res 2006; 98: 596-605.

31 Volpe M, Cosentino F, Tocci G, Palano F, Paneni F. Antihypertensive therapy in diabetes: the legacy effect and RAAS blockade. Curr Hypertens Rep 2011; 13: 318-324.

32 Ingelsson E, Sundstrom J, Arnlov J, Zethelius B, Lind L. Insulin resistance and risk of congestive heart failure. JAMA 2005; 294: 334-341. 